\title{
Nonlinear Approach to Thin-Walled Beams with a Symmetrical Open Section
}

\author{
Nina Andjelić* \\ Faculty of Mechanical Engineering, University of Belgrade, Serbia
}

\begin{abstract}
The principle of virtual work is applied to thin-walled beams with a cross-section with the middle line of an arbitrary curvilinear shape and with a continuously varying thickness. Six equilibrium equations and a seventh one related to the constrained torsion are derived taking into account general sectorial coordinates. The obtained relations are applied to structural elements with one longitudinal plane of symmetry with the shape similar to real turbine blades. All geometrical characteristics are calculated for one structural element with a modified cross-section shape. It has been shown that it is not recommendable to neglect the influence of secondary effects.

(C)2011 Journal of Mechanical Engineering. All rights reserved.
\end{abstract}

Keywords: nonlinear approach, general sectorial coordinates, curvilinear open sections, thin-walled beams, stresses

\section{INTRODUCTION}

Many modern metal structures (motor and railroad vehicles, naval structures, turbine blades) are manufactured using thin-walled elements (shells, plates, thin-walled beams). Thin-walled structures are applied when it is necessary to achieve high efficiency in strength and cost by minimizing the mass of the material used. Thin-walled beams are made from flat strips, manufactured by welding of thin plates or even machined, which permits a wide variety of crosssectional shapes.

Investigations of the behaviour of thinwalled members with open cross-sections have been carried out extensively since the early works of Timoshenko [1], Wagner [2] and Vlasov [3]. In addition to the mentioned authors, the theory of thin-walled structures was later developed by Kollbruner and Hajdin [4] and [5], Murray [6] and others all of whom showed that the cross-sections of thin-walled beams exhibit significant out-ofplane warping as a response to torsion.

It is well-known that both geometric nonlinearity and material inelasticity [7] are important for the investigation of the ultimate strength of thin-walled beams. The nonlinear analysis of beams and frames composed of thinwalled members has been generally carried out in the area of geometrical nonlinear elastic analysis. Early work on the large deflection and elastic analysis was presented by Powell [8] among others.

Nonlinear analysis can be defined as any analysis where linear extrapolation of stress, load and deflection is invalid [9] to [11]. Since the coupling between the nonlinear displacements and the rotational transformation relationships are complicated, certain approximations were usually done in order to simplify the derivations of the nonlinear strains. When approximations are made in the early stages of derivation, some significant terms of nonlinear strains can be lost. Open profiles are commonly analyzed by using Vlassov's assumption of vanishing of the warping shear strains in the middle surface of the thinwalled structure, and the warping of the profile is obtained as a function of the angle of rotation.

The linear theory of thin-walled members with open cross-sections, as presented in [4], differs from the conventional form by including the change of longitudinal normal stresses along the wall thickness. However, the basic differential equations (equilibrium conditions) are derived in the unique way. The linear "classical" theory of thin-walled open section beams was extended in [4] by including the secondary sectorial coordinates. That procedure is still linear and later in [12] the second order theory was extended to thin-walled members with an open cross-section with an arbitrary polygonal middle line.

*Corr. Author's Address: Faculty of Mechanical Engineering, University of Belgrade, 
A similar approach is applied in this paper to the sections with a middle line of an arbitrary curvilinear shape and with constantly varying thickness. The main purpose of this paper is to present an approach of a nonlinear analysis of beam-type structures with a thin-walled open cross-section. The authors tried to investigate the influence of the introduction of second order terms on the results.

\section{BASIC ASSUMPTIONS}

According to the classical linear theory of thin-walled open section beams [1], [3] and [4], normal stresses in cross-sections are assumed to be constant across the wall thickness and proportional to the sectorial coordinates. In [4] the secondary sectorial coordinate is introduced and the normal stresses are assumed to have the distribution that is not constant but linear across the wall thickness.

Basic assumptions of the theory of thinwalled beams are assumed to be valid:

(a) the cross-sections do not change their shape and their projections on the initial planes behave as rigid plates,

(b) the shear deformation in the middle surface is neglected,

(c) the line elements that are initially perpendicular to the middle surface remain straight and perpendicular during the whole deformation.

Open cross-sections are commonly analyzed by using Vlasov's assumptions: the warping shear strains are assumed to vanish in the middle surface of the thin-walled structure and the warping of the cross-section is obtained as a function of the angle of rotation.

\section{DISPLACEMENTS AND DEFORMATIONS}

Applying the principle of virtual work [12] and [13] to the deformed configuration of the considered thin-walled beam, apart from the six common scalar equations, an additional seventh equation is obtained and it includes the relations between the bimoment $B$, Saint Venant's torque $M_{3}$, warping torque $M_{\omega}$ and internal forces.

\subsection{Position Vector of an Arbitrary Point}

Let us consider a thin-walled beam with an open cross-section (Fig. 1) and let us assume that: $X_{i} \quad(i=1,2,3)$ are two centroidal principal axes of the cross-section and the centroidal longitudinal axis of the considered beam respectively,

$\vec{i}_{i} \quad(i=1,2,3)$ are the unit vectors of $X_{i}$,

$s$ is the coordinate measured along the middle line of the cross-section,

$e \quad$ is the distance from the middle line.

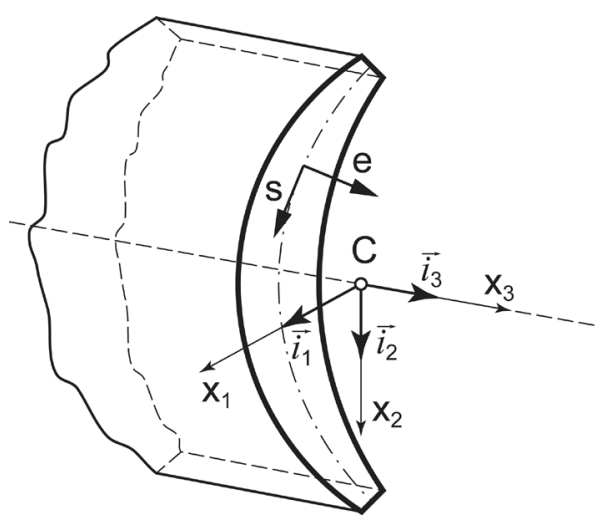

Fig. 1. Thin-walled open cross-section

Using the summation convention, the position of an arbitrary point in the cross-section (not in the middle surface) before the deformation is defined by:

$$
\vec{r}^{*}=x_{i}^{*} \cdot \vec{i}_{i}
$$

where $\vec{r}^{*}$ is position vector before the deformation, $x_{i}{ }^{*}(i=1,2,3)$ are material coordinates of an arbitrary point which is not initially in the middle surface.

\subsection{Displacements during the Deformation}

The position of an arbitrary point after the deformation can be defined by [12]:

$$
\vec{R}^{*}=\vec{r}^{*}+\vec{u}^{*}
$$

and by:

$$
\vec{R}^{*}=\left(x_{i}^{*}+u_{i}^{*}\right) \vec{i}_{i},
$$

where $\vec{R}^{*}$ is the position vector after the 
deformation, $\vec{u}^{*}$ is the displacement vector, $u_{i}^{*}$ $(i=1,2,3)$ are its components.

If $P$ is an arbitrary pole in the plane of the cross-section [4], starting from the assumptions about the deformations of the considered member, the components of the displacement vector can be obtained in the form:

$$
\begin{gathered}
u_{i}^{*}=\xi_{i P} \mp\left(x_{j}^{*}-x_{j P}\right) \phi_{P}(i, j=1,2 ; j \neq i), \\
u_{3}^{*}=-\xi_{1 P}^{\prime} x_{1}^{*}-\xi_{2 P}^{\prime} x_{2}^{*}-\phi_{P}^{\prime} \omega_{P}^{*}+w_{0},
\end{gathered}
$$

where $\xi_{i P}(i=1,2)$ are the displacement components of the pole $P$ in the directions of the axes $X_{i}(i=1,2), \varphi_{P}$ is the angle of rotation of the cross-section around the longitudinal axis through the pole $P, x_{i P}(i=1,2)$ are material coordinates of the pole $P, \omega_{P}{ }^{*}$ is the generalized sectorial coordinate, equal to the sum of the "classical" and the previously mentioned secondary sectorial coordinate, $w_{0}$ is the displacement in the direction of the longitudinal axis $X_{3} ;(\ldots)^{\prime}=d(\ldots) / d x_{3}$.

\subsection{Virtual Displacements}

If the virtual displacement

$$
\overline{\vec{u}}^{*}=\bar{u}_{i}^{*} \vec{i}_{i}
$$

is imposed to the points of the deformed beam, the position vector (3) will become

$$
\overrightarrow{\vec{R}}^{*}=\left(x_{i}^{*}+u_{i}^{*}+\bar{u}_{i}^{*}\right) \vec{i}_{i}
$$

and its projections on the directions of the axes $X_{i}$ $(i=1,2)$ are:

$$
u_{i}^{*}=\xi_{i P} \mp\left(x_{i}^{*}-x_{i P}\right) \phi_{P},
$$

where $\bar{u}_{i}^{*} \quad(i=1,2,3)$ are the components of the virtual displacement vector $\overline{\vec{u}}^{*}$.

\subsection{Virtual Deformations}

The assumption (b) about the shear deformation $\varepsilon_{s 3}$ in the middle surface gives as its consequence

$$
\begin{aligned}
\bar{\varepsilon}_{s 3} & =\overline{\vec{R}},{ }_{s} \overrightarrow{\vec{R}},_{3}= \\
& =\bar{u}_{i},{ }_{s}\left(x_{i}+u_{i}\right){ }_{3}+\bar{u}_{i},{ }_{3}\left(x_{i}+u_{i}\right),{ }_{s}=0,
\end{aligned}
$$

or, if developed,

$$
\begin{aligned}
-\bar{u}_{3, s}\left(1+u_{3,3}\right)= \\
=\bar{\phi}_{P}^{\prime}\left[x_{1} x_{2, s}-x_{2} x_{1, s}+x_{2 P} x_{1, s}-\right. \\
-x_{1 P} x_{2, s}+u_{1} x_{2, s}+x_{1} u_{2, s}+ \\
+u_{1} u_{2, s}+x_{2 P} u_{1, s}-u_{2} x_{1, s}- \\
\left.\quad-x_{2} u_{1, s}+u_{2} u_{1, s}-x_{1 P} u_{2, s}\right]+ \\
+\bar{\phi}_{P}\left[x_{2 P, s} x_{1,3}+x_{2 P, s} u_{1,3}+\right. \\
+x_{2 P, 3} x_{1, s}+x_{2 P, 3} u_{1, s}- \\
\quad-x_{1 P, s} x_{2,3}-x_{1 P, s} u_{2,3}- \\
\left.\quad-x_{1 P, 3} x_{2, s}-x_{1 P, 3} u_{2, s}\right]+ \\
+\bar{\xi}_{1 P}^{\prime}\left(x_{1}+u_{1}\right),_{s}+\bar{\xi}_{2 P}^{\prime}\left(x_{2}+u_{2}\right),_{s}
\end{aligned}
$$

where $\quad \overline{\vec{R}}=\left(x_{i}+u_{i}+\bar{u}_{i}\right), \quad x_{i} \quad(i=1,2,3)$ are coordinates of the middle surface, $u_{i}$ $(i=1,2,3)$ are the components of the displacement vector of the middle surface, $\bar{u}_{i}(i=1,2,3)$ are the components of the virtual displacement vector of the middle surface,

$$
\begin{gathered}
(\ldots)_{i}=\partial(\ldots) / \partial x_{i}, \quad(i=1,2), \\
(\ldots),_{e}=\partial(\ldots) / \partial e ; \quad(\ldots),_{s}=\partial(\ldots) / \partial s .
\end{gathered}
$$

Also, the consequence of the assumption (b) about the shear deformation $\varepsilon_{e 3}$ in the longitudinal plane perpendicular to the middle surface is:

$$
\begin{aligned}
\bar{\varepsilon}_{e 3} & =\overline{\vec{R}}^{*},{ }_{e} \cdot \overline{\vec{R}}^{*},_{3}= \\
& =\bar{u}_{i},_{e}^{*}\left(x_{i}^{*}+u_{i}^{*}\right),_{3}+\bar{u}_{i},_{3}^{*}\left(x_{i}^{*}+u_{i}^{*}\right),_{s}=0,
\end{aligned}
$$

or

$$
\begin{aligned}
-\bar{u}_{3, e}^{*}\left(1+u_{3,3}^{*}\right)= & \\
=\bar{\phi}_{P}[ & x_{1}{ }^{*} x_{2, e}{ }^{*}-x_{2}{ }^{*} x_{1, e}{ }^{*}+x_{2 P} x_{1, e}{ }^{*}- \\
& -x_{1 P} x_{2, e}+u_{1}{ }^{*} x_{2, e}{ }^{*}+x_{1}{ }^{*} u_{2, e}{ }^{*}+ \\
& +u_{1}{ }^{*} u_{2, e}{ }^{*}+x_{2 P} u_{1, e}{ }^{*}-u_{2}{ }^{*} x_{1, e}{ }^{*}- \\
& \left.-x_{2}{ }^{*} u_{1, e}{ }^{*}+u_{2}{ }^{*} u_{1, e}{ }^{*}-x_{1 P} u_{2, e}{ }^{*}\right]+ \\
+\bar{\phi}_{P}[ & x_{2 P, e} x_{1,3}{ }^{*}+x_{2 P, e} u_{1,3}{ }^{*}+ \\
& +x_{2 P, 3} x_{1, e}{ }^{*}+x_{2 P, 3} u_{1, e}{ }^{*}-x_{1 P, e} x_{2,3}{ }^{*}- \\
& \left.\quad x_{1 P, e} u_{2,3}{ }^{*}-x_{1 P, 3} x_{2, e}{ }^{*}-x_{1 P, 3} u_{2, e}{ }^{*}\right]+ \\
+ & \bar{\xi}_{1 P}\left(x_{1}{ }^{*}+u_{1}{ }^{*}\right)_{e}+\bar{\xi}_{2 P}\left(x_{2}{ }^{*}+u_{2}{ }^{*}\right)_{e}
\end{aligned}
$$




\section{EQUILIBRIUM CONDITIONS}

The equations of equilibrium are obtained using the expressions for the work of external and internal forces.

If $A$ is the cross-sectional area, if $\sigma_{3 i}$ $(i=1,2,3)$ are the stress components in the crosssection and if $p$ is the load acting over the middle surface of the considered beam, the virtual work $\bar{W}$ of external forces and $\bar{U}$ of internal forces [11] are:

$$
\begin{gathered}
\bar{W}=\int_{A}\left(\sigma_{3,3} \bar{u}^{*}+\sigma_{3} \bar{u}_{3,}^{*}\right) d A+\int_{s} \bar{p} \bar{u} d s, \\
\bar{U}=-\int_{A}\left(\sigma_{33} \bar{\varepsilon}_{33}^{*}+\sigma_{s 3} \bar{\varepsilon}_{s 3}^{*}\right) d A .
\end{gathered}
$$

Introducing real and virtual displacements and deformations into (14) and using the condition [12]

$$
\bar{W}+\bar{U}=0
$$

an equation of the following form is obtained:

$$
\begin{aligned}
& H_{1} w_{0}+H_{2} \xi_{1 P}+H_{3} \xi_{2 P}+H_{4} \phi_{P}+ \\
& +H_{5} \xi_{1 P}^{\prime}+H_{6} \xi_{2 P}^{\prime}+H_{7} \phi_{P}^{\prime}=0 .
\end{aligned}
$$

The functions $H_{i}(i=1,2, \ldots, 7)$ are defined by long expressions that are simplified if some of their parts are neglected:

$$
\begin{aligned}
H_{1}= & \int_{A}\left\{\sigma_{33}^{\prime}-\left[\sigma_{31} \xi_{1 P}^{\prime}+\sigma_{23} \xi_{2 P}^{\prime}-\right.\right. \\
& -\left(\sigma_{31}\left(x_{2}^{*}-x_{2 P}\right)-\right. \\
& \left.\left.\left.\left.-\sigma_{23}\left(x_{1}^{*}-x_{1 P}\right)\right) \phi_{P}^{\prime}\right]\right\}\right\} d A+ \\
& +\int_{s} \bar{p}_{3} d s, \\
H_{2}= & \int_{A}\left\{\sigma_{31}^{\prime}-\left[\sigma_{23} \phi_{P}-\right.\right. \\
& \left.\left.-\left(\xi_{1 P}^{\prime}-\phi_{P}^{\prime}\left(x_{2}^{*}-x_{2 P}\right)\right) \sigma_{33}\right]^{\prime}\right\} d A+ \\
& +\int_{s} \bar{p}_{1} d s, \\
H_{3}= & \int_{A}\left\{\sigma_{23}^{\prime}-\left[\sigma_{31} \phi_{P}-\right.\right. \\
& \left.\left.-\left(\xi_{2 P}^{\prime}+\phi_{P}^{\prime}\left(x_{1}^{*}-x_{1 P}\right)\right) \sigma_{33}\right]^{\prime}\right\} d A+ \\
& +\int_{s} \bar{p}_{2} d s,
\end{aligned}
$$

$$
\begin{aligned}
H_{4}=\int_{A}\left\{\sigma_{23}\left(x_{1}^{*}-x_{1 P}\right)-\sigma_{31}\left(x_{2}^{*}-x_{2 P}\right)+\right. \\
+\left[\xi_{1 P} \sigma_{23}-\xi_{2 P} \sigma_{31}-\right. \\
-\sigma_{33}\left(\xi_{1 P}^{\prime}\left(x_{2}^{*}-x_{2 P}\right)-\xi_{2 P}^{\prime}\left(x_{1}^{*}-x_{1 P}\right)\right)+ \\
\left.\left.+\phi_{P}^{\prime} \sigma_{33}\left(\left(x_{1}^{*}-x_{1 P}\right)^{2}+\left(x_{2}^{*}-x_{2 P}\right)^{2}\right)\right]\right\} d A+ \\
+\int_{S}\left\{\bar{p}_{2}\left(x_{1}-x_{1 P}\right)-\bar{p}_{1}\left(x_{2}-x_{2 P}\right)+\xi_{1 P} \bar{p}_{2}-\right. \\
\left.-\xi_{2 P} \bar{p}_{1}-\phi_{P}\left(\bar{p}_{1}\left(x_{1}-x_{1 P}\right)+\bar{p}_{2}\left(x_{2}-x_{2 P}\right)\right]\right\} d s, \\
H_{5}=\int_{A}\left\{\sigma_{33}^{\prime} x_{1}^{*}-\sigma_{31}-\right. \\
\quad-\phi_{P}\left[\sigma_{33}^{\prime}\left(x_{2}^{*}-x_{2 P}\right)-\sigma_{23}\right]+ \\
\left.\quad+\sigma_{33}^{\prime}\left(\xi_{1 P}+x_{2 P} \phi_{P}\right)+g^{\prime} x_{1}^{*}\right\} d A+ \\
\quad+\int_{s} \bar{p}_{3}\left[x_{1}-\phi_{P}\left(x_{2}-x_{2 P}\right)+\xi_{1 P}\right] d s, \\
H_{6}=\int_{A}\left[\sigma_{33}^{\prime} x_{2}^{*}-\sigma_{23}-\phi_{P}\left(\sigma_{33}^{\prime} x_{1}^{*}-\sigma_{31}\right)+\right. \\
+ \\
\left.+\sigma_{33}^{\prime}\left(\xi_{2 P}+x_{1 P} \phi_{P}\right)+g^{\prime} x_{2}^{*}\right] d A+ \\
\quad+\int_{s} \bar{p}_{3}\left[x_{2}+\phi_{P}\left(x_{1}-x_{1 P}\right)+\xi_{2 P}\right] d s, \\
H_{7}=\int_{A}\left[\sigma_{33}^{\prime} \omega_{P}^{*}-\sigma_{31}\left(x_{2}^{*}-x_{2 P}\right)-\sigma_{23}\left(x_{1}-x_{1 P}\right)+\right. \\
+2 \sigma_{s 3} \phi_{P}^{\prime} e+ \\
+\xi_{1 P}\left(\sigma_{33}^{*} x_{2}^{*}-\sigma_{23}\right)-\xi_{2 P}\left(\sigma_{33}^{\prime} x_{1}^{*}-\sigma_{31}\right)- \\
\left.-\sigma_{33}^{\prime}\left(\xi_{1 P} x_{2 P}-\xi_{2 P} x_{1 P}\right)+g^{\prime} \omega_{P}^{*}\right] d A+ \\
+\int_{p_{3}}\left[\omega_{P}+\xi_{1 P}\left(x_{2}-x_{2 P}\right)-\xi_{2 P}\left(x_{1}-x_{1 P}\right)\right] d s,
\end{aligned}
$$

where:

$$
g=\sigma_{31}\left[\xi_{1 P}^{\prime}-\phi_{P}\left(x_{2}^{*}-x_{2 P}\right)\right]+\sigma_{23}\left[\xi_{2 P}^{\prime}+\phi_{P}^{\prime}\left(x_{1}-x_{1 P}\right)\right]
$$

In order to satisfy the Eq. (16), the Eq. (17) to (23) must equal zero. After the integration over the cross-sectional area [12] the following Eqs. are obtained:

$$
\begin{aligned}
& F_{3}^{\prime}-\left(F_{1} \xi_{1 P}^{\prime}+F_{2} \xi_{2 P}^{\prime}+M_{3} \phi_{P}^{\prime}\right)^{\prime}+p_{3}=0 \\
& F_{1}^{\prime}+\left[F_{3}\left(\xi_{1 P}^{\prime}+x_{2 P} \phi_{P}^{\prime}\right)-M_{2} \phi_{P}^{\prime}-F_{2} \phi_{P}\right]^{\prime}+p_{1}=0 \\
& F_{2}^{\prime}+\left[F_{3}\left(\xi_{2 P}^{\prime}+x_{1 P} \phi_{P}^{\prime}\right)+M_{1} \phi_{P}^{\prime}+F_{1} \phi_{P}\right]^{\prime}+p_{2}=0 \\
& M_{3}^{\prime}+\left[F_{2} \xi_{1 P}-F_{1} \xi_{2 P}-\xi_{1 P}^{\prime}\left(M_{2}-F_{3} x_{2 P}\right)+\right. \\
& +\xi_{2 P}^{\prime}\left(M_{1}-F_{3} x_{1 P}\right)+ \\
& \left.+\phi_{P}^{\prime}\left(F_{3} i_{P}^{2}+M_{1} \beta_{1}+M_{2} \beta_{2}+B \beta_{\omega}\right)\right]^{\prime}+ \\
& +m_{3}+p_{2} \xi_{1 P}-p_{1} \xi_{2 P}-\phi_{P} m_{3}=0,
\end{aligned}
$$




$$
\begin{aligned}
& M_{1}^{\prime}-F_{1}+m_{1}-\phi_{P}\left(M_{2}^{\prime}-F_{2}+m_{2}\right)+ \\
& +\left(F_{3}^{\prime}+p_{3}\right)\left(\xi_{1 P}-x_{2 P} \phi_{P}\right)+G_{1}=0, \\
& M_{2}^{\prime}-F_{2}+m_{2}+\phi_{P}\left(M_{1}^{\prime}-F_{1}+m_{1}\right)+ \\
& +\left(F_{3}^{\prime}+p_{3}\right)\left(\xi_{2 P}-x_{1 P} \phi_{P}\right)+G_{2}=0, \\
& B^{\prime}-M_{\omega}+b_{\omega}+\xi_{1 P}\left(M_{2}^{\prime}-F_{2}+m_{2}\right)- \\
& -\xi_{2 P}\left(M_{1}^{\prime}-F_{1}+m_{1}\right)- \\
& -\left(F_{3}^{\prime}+p_{3}\right)\left(\xi_{1 P} x_{2 P}-\xi_{2 P} x_{1 P}\right)+G_{\omega}=0 .
\end{aligned}
$$

Seven Eqs. of equlibrium are obtained and the seventh one (31) is the Eq. connected with the constrained torsion.

In the Eqs. (25) to (31) the following notations are introduced:

- $F_{i}(i=1,2,3)$ are two transversal forces and the axial force respectively,

- $\quad M_{i}$ and $m_{i}(i=1,2)$ are concentrated and continuously distributed bending moments,

- $\quad M_{3}$ and $m_{3}$ are concentrated and continuously distributed torques about the longitudinal axis (Saint Venant's torques):

$$
M_{3}=\int_{A}\left[\sigma_{23}\left(x_{1}^{*}-x_{1 P}\right)-\sigma_{31}\left(x_{2}^{*}-x_{2 P}\right)\right] d A,
$$

- $\quad M_{\omega}$ and $m_{\omega}$ are concentrated and continuously distributed warping moments,

- $\quad B$ and $b_{\omega}$ are concentrated and continuously distributed bimoments defined through the general sectorial coordinates,

- $G_{i}(i=1,2,3), \beta_{i}(i=1,2), \beta_{\omega}$ and $i_{P}$ are:

$$
\begin{gathered}
G_{1}=\int_{A} g^{\prime} x_{1}^{*} d A, \\
G_{2}=\int_{A} g^{\prime} x_{2}^{*} d A, \\
G_{\omega}=\int_{A} g^{\prime} \omega_{P}^{*} d A, \\
\beta_{1}=\frac{1}{J_{2}} \int_{A} x_{1}^{*}\left(x_{1}^{* 2}+x_{2}^{* 2}\right) d A-2 x_{1 P}, \\
\beta_{2}=\frac{1}{J_{2}} \int_{A} x_{2}^{*}\left(x_{1}^{* 2}+x_{2}^{*_{2}}\right) d A-2 x_{2 P}, \\
\beta_{2}=\frac{1}{J_{2}} \int_{A} x_{2}^{*}\left(x_{1}^{* 2}+x_{2}^{* 2}\right) d A-2 x_{2 P}, \\
i_{P}^{2}=x_{1 P}^{2}+x_{2 P}^{2}+\frac{1}{A}\left(J_{1}+J_{2}\right),
\end{gathered}
$$

- $J_{i}$ are the principal centroidal moments of inertia of the cross-section

$$
J_{i}=\int_{A} x_{j}^{* 2} d A, \quad(i, j=1,2 ; \quad i \neq j),
$$

- $J_{\omega}$ is the generalized sectorial moment of inertia of the cross-section

$$
J_{\omega}=\int_{A} \omega_{P}^{* 2} d A
$$

The quantities (33) to (41) are calculated taking into account the secondary effects.

If the expressions are linearized, the Eqs. (17) to (23) are reduced to the Eqs. of the linear theory of thin-walled beams shown in [1], [3] and [4].

\section{NUMERICAL EXAMPLE}

The real steam turbine blades usually have the cross-sections of the shapes shown in Fig. 2 [14] and they have to be treated as thin-walled sections of non-constant thickness.

The real cross-sections (Fig. 2) were approximated for the calculations by the crosssection with one axis of symmetry (Fig. 3).

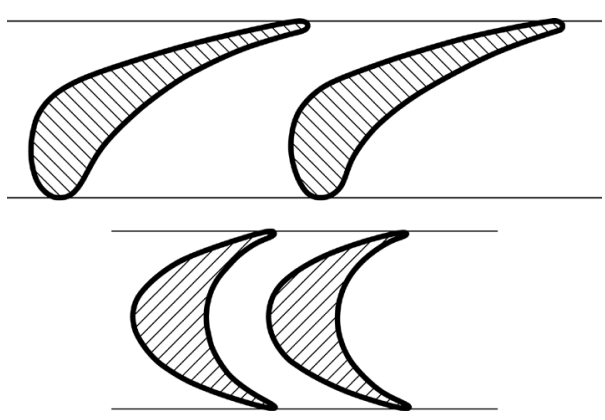

Fig. 2. Real cross-sections

In order to apply the derived equations and expressions to the elements with cross-sections similar to the cross-sections of the real turbine blades, one element of the modified shape of the cross-section with one axis of symmetry (Fig. 3 ) was chosen for the calculations. Its symmetry obviously induced some simplifications in the used expressions, and the whole element was considered as clamped at one end and loaded in a complex way by continuously distributed lateral load and torque along its length. 


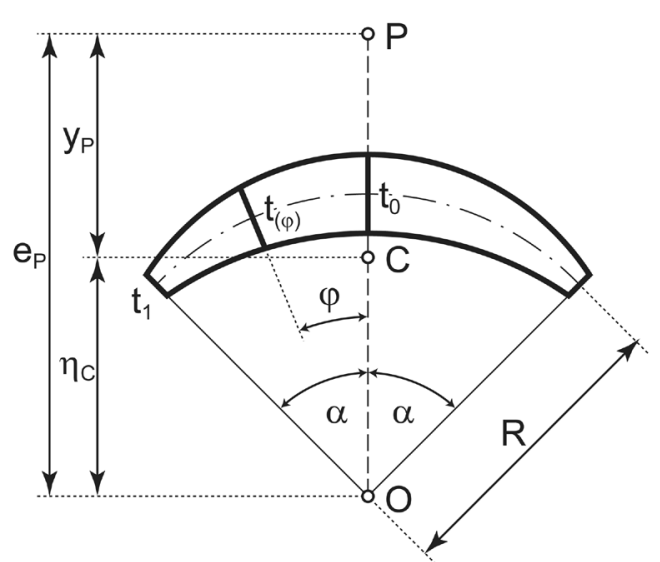

Fig. 3. Approximated cross-section; $R$ is the radius of curvature of the middle line; $e_{P}$ is the distance between the center of curvature $O$ and the pole $P$ (shear center); $\eta_{C}$ is the distance between the center of curvature $O$ and the centroid of the cross-section $C ; y_{P}=e_{P}-\eta_{C}$

A certain problem presented the choice of the function by which the variation of the thickness along the section could be described. The chosen cross-section having non-constant thickness $t(\varphi)$ (Fig. 3) was defined by the following Eq. [15].

$$
t(\phi)=t_{0}-\left(t_{0}-t_{1}\right) \phi / \alpha
$$

where $t_{0}=t(\phi=0), t_{1}=t(\phi=\alpha)$.

\subsection{Geometrical Characteristics}

The expressions defining the geometrical characteristics needed for the calculation were derived applying the function (42):

- Cross-sectional area

$$
A=R t_{0} \alpha\left(1+t_{1} / t_{0}\right)
$$

- Torsion constant

$$
\begin{aligned}
I_{t} & =(1 / 3) \int_{\phi} t(\phi)^{3} R d \phi= \\
& =\left(R t_{0}^{3} \alpha / 6\right)\left[1+t_{1} / t_{0}+\left(t_{1} / t_{0}\right)^{2}+\left(t_{1} / t_{0}\right)^{3}\right],
\end{aligned}
$$

- Generalized sectorial moment of inertia

$J_{\omega} / R^{5} t_{0}=\left(\alpha^{3} / 6\right)\left(1+3 t_{1} / t_{0}\right)+$

$+4 e_{P} / R\left\{(1 / \alpha)\left(1-t_{1} / t_{0}\right)\left(\alpha \sin \alpha+2 \cos \alpha-2-\left(e_{P} / 8 R\right) \sin ^{2} \alpha\right)+\left(t_{1} / t_{0}\right)\left(1+t_{0}^{3} / 12 t_{1} R^{2}\right)(\alpha \cos \alpha-\sin \alpha)+\right.$

$+\left(e_{P} / 8 R\right)\left[\left(1+t_{1} / t_{0}\right) \alpha-\left(t_{1} / t_{0}\right) \sin 2 \alpha\right]+\left(t_{0} / 2 R\right)^{2}\left[-(1 / \alpha)\left(1-t_{1} / t_{0}\right)\left(\alpha^{2} \cos \alpha-2 \alpha \sin \alpha-2 \cos \alpha+2\right)+\right.$

$+\left(1 / \alpha^{2}\right)\left(1-t_{1} / t_{0}\right)\left(\alpha^{3} \cos \alpha-3 \alpha^{2} \sin \alpha-6 \alpha \cos \alpha+6 \sin \alpha\right)-(4 / 3)\left(1 / \alpha^{3}\right)\left(1-t_{1} / t_{0}\right)^{3}\left(\left(\alpha^{4} / 4\right) \cos \alpha-\alpha^{3} \sin \alpha\right.$

$\left.\left.\left.\left.-3 \alpha^{2} \cos \alpha+6 \alpha \sin \alpha+6 \cos \alpha-6\right)\right]\right\}+t_{0} / 2 R\right)^{2}\left(e_{P} / R\right)^{2}[\alpha-\sin \alpha \cos \alpha-3 / 2 \alpha)\left(1-t_{1} / t_{0}\right)\left(\alpha^{2}-\alpha \sin 2 \alpha+\sin ^{2} \alpha\right)+$ $+\left(3 / 2 \alpha^{2}\right)\left(1-t_{1} / t_{0}\right)^{2} \cdot\left(2 \alpha^{3} / 3-\alpha^{2} \cdot \sin 2 \alpha-\alpha+2 \alpha \sin ^{2} \alpha+\sin \alpha \cos \alpha\right)-\left(3 / 4 \alpha^{3}\right)\left(1-t_{1} / t_{0}\right)^{3}\left(\alpha^{4} / 3-\left(2 \alpha^{3} / 3\right) \sin 2 \alpha+\right.$ $\left.\left.+2 \alpha^{2} \sin ^{2} \alpha-\alpha^{2}+\alpha \sin 2 \alpha-\sin ^{2} \alpha\right)\right]$.

- After the integration the quantities $\beta_{1}, \beta_{2}$ and $\beta_{\omega}$ defined by (36) to (38) become:

$$
\begin{aligned}
& \beta_{1} /\left(R^{2} t_{0}^{3} / 48 J_{2}\right)=12\left[1+4 \rho_{0}^{2}\left(1+\eta^{2}\right)\right](1-\cos \alpha)-4 \eta\left(1+12 \rho_{0}^{2}\right) \sin ^{2} \alpha- \\
& -12\left(1-t_{1} / t_{0}\right)(1 / \alpha)\left[3+4 \rho_{0}^{2}\left(1+\eta^{2}\right)(\sin \alpha-\alpha \cos \alpha)+\eta\left(1+\rho_{0}^{2}\right)\left(\alpha-\sin \alpha \cos \alpha-2 \alpha \sin ^{2} \alpha\right)\right]- \\
& -6\left(1-t_{1} / t_{0}\right)^{2}\left(1 / \alpha^{2}\right)\left[6\left(2-2 \alpha \sin \alpha-2 \cos \alpha+\alpha^{2} \cos \alpha\right)-\eta\left(\alpha^{2}-\alpha \sin 2 \alpha+\sin ^{2} \alpha-2 \alpha^{2} \sin ^{2} \alpha\right)\right]+ \\
& +3\left(1-t_{1} / t_{0}\right)^{3}\left(1 / \alpha^{3}\right)\left\{12\left(2 \sin \alpha-2 \alpha \cos \alpha-\alpha^{3} \sin \alpha+\alpha^{3} \cos \alpha\right)+\eta\left[\alpha\left(3-2 \alpha^{2}\right)\left(1-2 \sin ^{2} \alpha\right)\right.\right. \\
& \left.\left.-3\left(1-2 \alpha^{2}\right) \sin \alpha \cos \alpha\right]\right\},
\end{aligned}
$$

$\beta_{2} /\left(R^{2} t_{0}^{3} / 48 J_{1}\right)=\left\{12\left[1+4 \rho_{0}^{2}\left(1+\eta^{2}\right)\right]-4 \eta\left(1+12 \rho_{0}^{2}\right)(\alpha+\sin \alpha \cos \alpha)-4 \alpha \eta\left[1+12 \rho_{0}^{2}\left(1+3 \eta^{2}\right)\right]+\right.$

$+12\left(1-t_{1} / t_{0}\right)(1 / \alpha)\left[3+4 \rho_{0}^{2}\left(1+3 \eta^{2}\right)(1-\cos \alpha-\alpha \sin \alpha)+\eta\left(1+4 \rho_{0}^{2}\right)\left(\alpha^{2}+2 \alpha \sin \alpha \cos \alpha-\sin ^{2} \alpha\right)\right.$

$\left.-\eta\left(1+4 \rho_{0}^{2}\right) \alpha^{2}\right]+2\left(1-t_{1} / t_{0}\right)^{2}\left(1 / \alpha^{2}\right)\left[18\left(\alpha^{2} \sin \alpha+2 \cos \alpha-2 \sin \alpha\right)-\right.$$$
\left.-\eta\left(4 \alpha^{3}+6 \alpha^{2} \sin \alpha \cos \alpha-3 \alpha \sin ^{2} \alpha+3 \alpha-3 \sin \alpha \cos \alpha\right)\right]-\left(1-t_{1} / t_{0}\right)^{3}\left(1 / \alpha^{3}\right)\left[12\left(\alpha^{3} \sin \alpha+3 \alpha^{2} \cos \alpha-6 \alpha \sin \alpha-6 \cos \alpha+6\right)-\right.
$$$$
\left.\left.-2 \alpha^{4}+4 \alpha^{3} \sin \alpha \cos \alpha-3 \alpha^{2}\left(2 \sin ^{2} \alpha-1\right)-6 \alpha \sin \alpha \cos \alpha+3 \sin ^{2} \alpha\right]\right\}-y_{P}\left(R^{2} t_{0}^{3} / 48 J_{1}\right) \text {, }
$$ 


$$
\begin{aligned}
& \beta_{\omega} /\left(R^{3} t_{0} / 120 J_{\omega}\right)=10\left[1+12 \rho_{0}^{2}\left(1+\eta^{2}\right) \alpha^{2}\right]+20\left(e_{P} / R\right)\left[1-12 \rho_{0}^{2}\left(1+\eta^{2}\right)\right](1-\cos \alpha)+ \\
& +480 \rho_{0}^{2} \eta(1-\alpha \sin \alpha-\cos \alpha)-20\left(e_{P} / R\right) \eta\left(1+12 \rho_{0}^{2}\right) \sin ^{2} \alpha-5\left(1-t_{1} / t_{0}\right)(1 / \alpha)\left\{\left[4+16 \rho_{0}^{2}\left(1+\eta^{2}\right)\right] \alpha^{3}-\right. \\
& -96 \rho_{0}^{2} \eta\left(2 \alpha \cos \alpha-\sin \alpha+\alpha^{2} \sin \alpha\right)+12\left(e_{P} / R\right)\left[1-4 \rho_{0}^{2}\left(1+\eta^{2}\right)\right](\alpha \cos \alpha-\sin \alpha)+ \\
& \left.+6\left(e_{P} / R\right) \eta\left(1+4 \rho_{0}^{2}\right)(\alpha-2 \alpha \sin \alpha-\sin \alpha \cos \alpha)\right\}-15\left(1-t_{1} / t_{0}\right)^{2}\left(1 / \alpha^{2}\right)\left[8-8 \alpha \sin \alpha-8 \cos \alpha+4 \alpha^{2} \cos \alpha+\right. \\
& \left.+2\left(e_{P} / R\right) \eta\left(2 \alpha \sin \alpha \cos \alpha-\alpha^{2}+2 \alpha^{2} \sin ^{2} \alpha-\sin ^{2} \alpha\right)-\alpha^{4}\right]- \\
& -\left(1-t_{1} / t_{0}\right)^{3}\left(1 / \alpha^{3}\right)\left\{20\left(e_{P} / R\right)\left(6 \alpha \cos \alpha-6 \sin \alpha+3 \alpha^{2} \sin \alpha-\alpha^{3} \cos \alpha\right)+\right. \\
& \left.+5\left(e_{P} / R\right)\left[\left(1-2 \sin ^{2} \alpha\right)\left(3 \alpha-2 \alpha^{3}\right)-3\left(1-2 \alpha^{2}\right) \sin \alpha \cos \alpha\right]+4 \alpha^{5}\right\},
\end{aligned}
$$

where $\eta=\eta_{\mathrm{C}} / \mathrm{R}, \rho_{0}=R / t_{0}$.

It must be underlined that according to the author's knowledge the Eq. (45) for the generalized sectorial moment of inertia and Eqs. (46) to (48) are for the first time derived in this paper.

\subsection{Stress Distribution}

Stress components that appear in the considered case are normal and shear stresses caused by bending and torsion. Attention is particularly paid to the constrained torsion effects as well as to the stress components, which are their consequences as in that case the secondary effects become evident.

Normal stresses are proportional to the sectorial coordinate and it is necessary to point out that the secondary sectorial coordinate will be taken into consideration together with the generalized sectorial moment of inertia calculated using (41).

Normal stresses $\sigma$ caused by the bimoment and shear stresses $\tau$ caused by the torsion [4] and [15] are calculated from the Eqs. (49) and (50):

$$
\begin{gathered}
\sigma=\frac{B}{J_{\omega}} \omega, \\
\tau=\frac{M_{3}}{I_{t}} t,
\end{gathered}
$$

where $t$ - is the thickness of the cross-section at the considered place.

As the shear stresses and particularly stresses induced by the Saint Venant's torques should not be neglected, it was necessary to calculate the equivalent stresses $\sigma_{e}$, which was done using the maximum shear stress failure (Tresca) criterion (51)

$$
\sigma_{e}=\sqrt{\sigma^{2}+4 \tau^{2}}
$$

The calculations were done for the straight beam (Fig. 4) with the chosen cross-section (Fig. 3), and for the dimensions: $L=900 \mathrm{~mm}$, $R=300 \mathrm{~mm}, \alpha=30^{0}, t_{0}=26 \mathrm{~mm}, t_{1}=13 \mathrm{~mm}$.

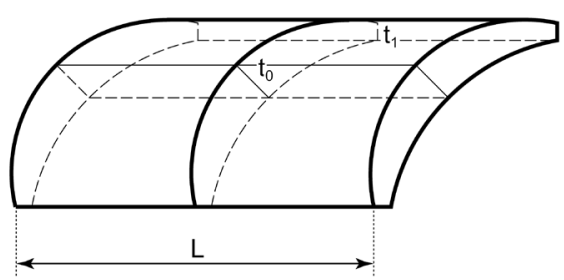

Fig. 4. Approximated shape of the steam turbine blade

As the aim of the paper is to investigate whether it is necessary or not to take into account the additional effects, secondary effects and sectorial coordinates, the stresses were calculated for the unit loads.

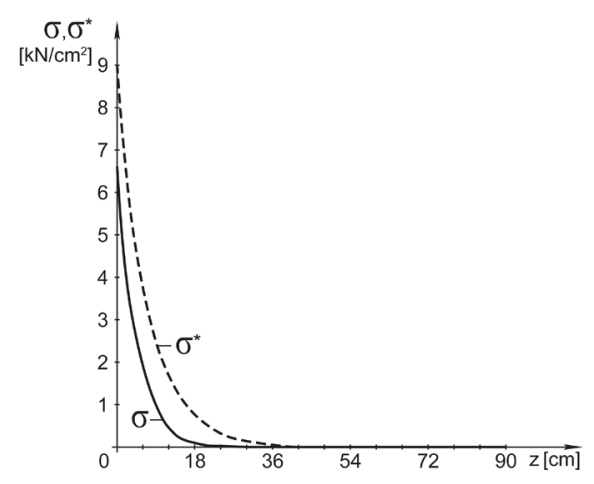

Fig. 5. Distribution of normal stresses

The distribution of maximal stresses along the beam is presented in Fig. 5 for normal stresses, in Fig. 6 for shear stresses, and in Fig. 7 for equivalent stresses. Numerical calculations were 
performed separately for the "classical" case when the secondary sectorial coordinate was not taken into account.

The stress values connected with secondary effects are denoted by asterisk.

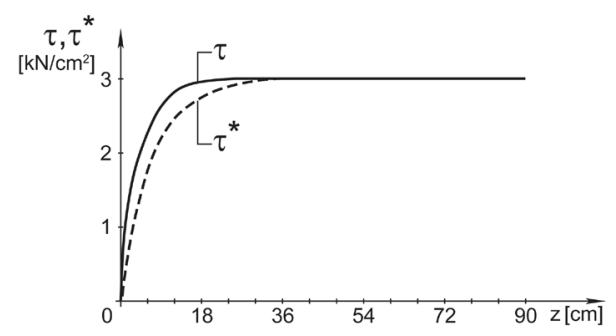

Fig. 6. Distribution of shear stresses

\section{CONCLUSIONS}

All geometrical characteristics for the chosen cross-section and, after that, all stress components for the considered structural element having the shape of the turbine blade were calculated in the way explained in [4], [13] and [15]. As it has been mentioned the Eqs. (45) to (48) are for the first time derived in this paper. The geometrical quantities obtained with and without the secondary sectorial coordinates were mutually compared as well as the stress components in the case of complex loads. Their distributions over the cross-section and along the element for the applied unit loads were determined. The dependence of the secondary effects on the crosssectional dimensions was shown.

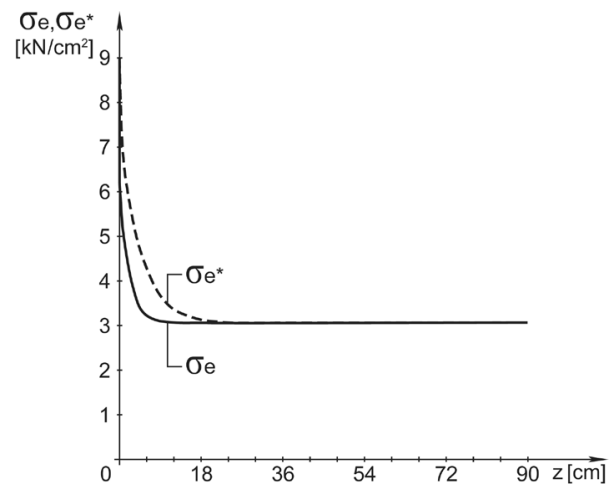

Fig. 7. Distribution of equivalent stresses
From the obtained results it can be concluded that if secondary effects are taken into account, the differences between the stresses with and without the mentioned effects are quite remarkable at the clamped ends and that they decrease towards the middle of the beam. It should be pointed out that the clamped ends are at the same time the places where the blades are connected with the rotor body and that they already represent the critical points.

If the additional terms are taken into account, they have non negligible effects on the level of stress components, particularly in some cases of the crotional shapes similar to those considered in the numerical example.

\section{REFERENCES}

[1] Timoshenko, S.P. (1945). Theory of bending, torsion and buckling of thin-walled members of open cross section. Journal of the Franklin Institute, vol. 239, no. 3, p. 201-219, vol. 239, no. 4, p. 249-268, vol. 239, no. 5, p. 343-361.

[2] Wagner, H., Pretschner, W. (1934). Verdrehung und Knickung von offenen Profilen. Luftfahrtforschung, vol. 11, no. 6, p. 174-180.

[3] Vlasov, V.Z. (1959). Thin-walled elastic beams, $2^{\text {nd }}$ ed. Moscow (English translation, Jerusalem, 1961).

[4] Kollbruner, C.F., Hajdin, N. (1972). Dunnwandige Stabe, Band 1. Springer Verlag, Berlin.

[5] Kollbruner, C.F., Hajdin, N. (1975). Dunnwandige Stabe, Band 2. Springer Verlag, Berlin.

[6] Murray, N.W. (1984). Introduction to the theory of thin-walled Structures. Clarendon Press, Oxford.

[7] Epstein, M., Murray, D.W. (1996). Threedimensional large deformation analysis of thin-walled beams. International Journal of Solids and Structures, vol. 12, no. 12, p. 867876.

[8] Powell, G.H. (1969). Theory of nonlinear elastic structures. Journal of the Structural Division, ASCE, vol. 95 , no. 12, p. 26872701.

[9] Chan, S.L. (2001). Non-linear behaviour and design of steel structures. Journal of 
Constructional Steel Research, vol. 57, no. 12, p. 1217-1231.

[10] Sekulovic, M. (1986). Geometrically nonlinear analysis of thin-walled members, steel struct., recent 'research advances and their applications to design'. Elsevier Applied Science Publishers, part 12.

[11] Harl, B., Kegl, M. (2005). Efficient shape optimization of space trusses. Strojniški vestnik - Journal of Mechanical Engineering vol. 51 , no. 9 , p. $570-588$.

[12] Hajdin, N. (1982). The non-linear theory of thin-walled member with open cross section. Theoretical and Applied Mechanics, vol. 8, p. 29-39.

[13] Erkmen, R.E., Bradford, M.A. (2009). Nonlinear elasto-dynamic analysis of I-beams curved in-plan. International Journal of Structural Stability and Dynamics, vol. 9, no. 2, p. 213-242.

[14] Vasiljević, N. (1990). Steam Turbines. University of Belgrade, Faculty of Mechanical Engineering, Belgrade. (in Serbian)

[15] Rnjak, N. (1993). A contribution to the analysis of behaviour of thin-walled structural elements of turbine blade shapes, MSci Thesis. University of Belgrade, Faculty of Mechanical Engineering, Belgrade. (in Serbian)

[16] Ružić, D. (1995). Strength of Structures. Universityof Belgrade, Faculty of Mechanical Engineering, Belgrade. (in Serbian) 